\title{
¿Qué sabe el alumnado sobre las problemáticas socio- ambientales del agua y su gestión sostenible? Investigación mixta en Educación Primaria
}

\author{
María-Paz Pozo-Muñoz \\ Facultad de Ciencias de la Educación, Universidad de Málaga. Málaga. España.maria.paz:pozo@uma.es \\ Leticia-Concepción Velasco-Martínez iD \\ Departamento de Investigación y Psicologia en Educación, Facultad de Educación, Universidad Complutense \\ de Madrid.Madrid.España.letivela@ucm.es \\ Carolina Martín-Gámez (D) \\ Departamento de Didáctica de la Matemática, de las Ciencias Sociales y de las Ciencias Experimentales, \\ Universidad de Málaga.Málaga. España.cmarting@uma.es \\ Juan-Carlos Tójar-Hurtado iD \\ Departamento de Teoría e Historia de la Educación y Métodos de Investigación y Diagnóstico en Educación, \\ Universidad de Málaga.Málaga.España.jctojar@uma.es
}

[Recibido: 14 octubre 2020. Revisado: 9 noviembre 2020. Aceptado: 24 enero 2021]

Resumen: El agua como contenido curricular siempre ha recibido una gran atención por la comunidad educativa, no solo por su interés y valor educativo, sino también por las dificultades de aprendizaje detectadas durante su enseñanza. Las últimas investigaciones revelan que el alumnado tiene problemas para identificar por dónde se mueve el agua en la naturaleza o de dónde viene el agua del grifo hasta sus viviendas. Este estudio analiza los conocimientos científicos que tienen los estudiantes de tercer ciclo de Educación Primaria respecto del ciclo hidrológico y las causas de sus problemáticas socioambientales. Desde un enfoque de investigación mixto (CUAL $\rightarrow$ cuan), se analiza el contenido de cartas y dibujos elaboradas por 95 estudiantes de un centro educativo de Málaga. Los resultados de este trabajo muestran los retos que plantea el aprendizaje de los conceptos relacionados con el agua y sus implicaciones educativas.

Palabras clave: Agua; Conocimiento previo; Ciclo hidrológico; Gestión de los recursos hídricos; Contaminación del agua; Educación Primaria.

What do students know about the socio-environmental problems of water and its sustainable management? Mixed Research in Primary Education

\begin{abstract}
Water as a curricular content has always received great attention from the educational community, not only for its interest and educational value, but also for the learning difficulties detected during its teaching. The latest research reveals that students have trouble identifying where water in nature moves or where tap water comes from to their homes. This study analyzes the scientific concepts that Primary Education third cycle students have regarding the hydrological cycle and the causes of their socio-environmental problems. From a mixed research approach (QUAL $\rightarrow$ quan), the content of letters and drawings prepared by 95 students from an educational center in Malaga is analyzed. The results of this work show the challenges posed by learning the concepts related to water and their educational implications.
\end{abstract}

Keywords: Water, Previous knowledge; Hydrological cycle; Management of water resources, Pollution water; Primary Education.

Para citar este artículo: Pozo-Muñoz M. P., Velasco-Martínes L. C., Martín-Gámez C. y Tójar-Hurtado J. C. (2021) ¿Qué sabe el alumnado sobre las problemáticas socio-ambientales del agua y su gestión sostenible? Investigación mixta en Educación Primaria. Revista Eureka sobre Enseñanza y Divulgación de las Ciencias 18(3), 3501. doi: 10.25267/Rev_Eureka_ensen_divulg_cienc.2021.v18.i3.3501 


\section{Introducción}

Según el Real Decreto 126/2014, de 28 de febrero, de la Educación Primaria, los contenidos de ciencias se estructuran alrededor de algunos conceptos fundamentales cuyo tratamiento permite a los estudiantes avanzar en la construcción del conocimiento científico de manera articulada y coherente. Para ello, su enseñanza debe partir del conocimiento previo que posee el alumnado al llegar al aula (Furió, Solbes y Carrascosa 2006), dado que supone un punto de partida para conocer su manera de comprender y adquirir nuevos conocimientos científicos (Driver, Guesne y Tiberghein 1985). Este hecho pone de relieve el interés creciente que desde la didáctica de las ciencias se ha mostrado en torno al estudio de los conocimientos iniciales del alumnado sobre diversas temáticas (Amahmid et al. 2019; Basterretxea, Bastida, Díez y Gutiérrez 2019; Durkan, Güngör, Fetihi, Erol y Ogelman 2016; Karpudewan, Roth y Abdullah 2015; Malandrakis y Chatzakis 2014; Mora 2017; Sari, Karkkainen y Tuula 2018).

Más concretamente, en relación directa con el tema del agua, Fernández-Arroyo y Rodríguez Marín (2017) sostienen que los conceptos como el ciclo natural y urbano, los tipos de usos del agua y la contaminación, son los "contenidos prototípicos" relacionados con esta temática que más se abordan dentro de los estudios sobre las ideas alternativas de los estudiantes. En este sentido, autores como Cañal, García-Carmona y Cruz-Guzmán (2016) y Díez (2017) advierten que estos conceptos sobre el agua reciben tanta atención e interés por las dificultades de aprendizaje que se detectan durante su enseñanza, los cuales señalan que deben ser abordados desde una perspectiva educativa centrada en el estudiante y basada en el aprendizaje significativo.

Por tanto, el objetivo principal del estudio se dirige a conocer y analizar los conocimientos científicos que tienen los estudiantes de Educación Primaria (EP, en adelante) sobre los procesos naturales, el uso y gestión del agua, y las problemáticas derivadas de la escasez y la contaminación de esta. Además, este trabajo persigue contribuir a mejorar los programas educativos y ampliar las posibilidades de actuación del profesorado en relación con este contenido curricular.

\section{Conocimientos científicos sobre el concepto de agua}

Los estudios sobre los conocimientos científicos de los estudiantes sobre el concepto de agua y su posible tratamiento dentro del ámbito curricular en la EP, vienen desarrollándose desde hace varios años. Así, se encuentran los primeros trabajos centrados en el ciclo del agua que advierten que los estudiantes de EP sitúan el origen del ciclo en las nubes (Bar 1989; Massa 1994; Taiwo, Ray, Motswiri y Masene 1999). Estudios ya más recientes indican que el conocimiento que tiene la población sobre el ciclo del agua no ha avanzado todo lo deseado y, por ejemplo, desconocen por dónde se mueve el agua en la naturaleza o de dónde viene el agua del grifo de sus viviendas (Díez, 2017). En este sentido, Guerrero Valle (2017) puso de manifiesto que suele ser común que los estudiantes de EP crean que el ciclo comienza en otros lugares como el mar o los montes, e identifiquen los sistemas y procesos que intervienen en el mismo, pero no lo comprendan de manera integral (De Miguel et al. 2009). También, Martín del Pozo (2013) señala que existe un número muy limitado de estudiantes que llega a comprender y representar el ciclo del agua de manera completa, es decir, considerando su carácter cerrado y cíclico. Esta falta de entendimiento del ciclo del agua como un circuito cerrado supone, según Cañal, García-Carmona y Cruz-Guzmán (2016), un impedimento para tomar conciencia sobre los problemas de escasez de agua y su necesidad de ahorro.

En esta línea, Covitt, Gunckel y Anderson (2009) pusieron de manifiesto que para llegar a adquirir conocimientos medioambientales, es necesario comprender la estructura de los sistemas a través de los cuales fluye el agua, y conocer cómo circula la materia (agua y otras 
sustancias) a través de los sistemas naturales (cambios de estado y soluciones) y a través de las infraestructuras humanas (sistemas de distribución, saneamiento y depósitos de agua). Sin embargo, estos autores descubrieron que la mayoría de los estudiantes de EP desconocían los movimientos y cambios de estado del agua (y de otros materiales) que se producen a través de los sistemas. De hecho, tenían muchas dificultades para percibir los aspectos menos visibles que se dan en los ecosistemas hídricos a escala micro (atómica-molecular) y macro (configuración del paisaje). De la misma manera, Martín del Pozo (2013) indicaba que en el tercer ciclo de EP, el 90\% de los estudiantes reconocían el paso de líquido a gas, mientras en el segundo ciclo solo lo hace un $60 \%$ aludiendo a que "el agua se va a la atmósfera", "se evapora" o "se va a las nubes". Respecto del proceso de condensación, esta autora también señala que la percepción de los estudiantes queda restringida a la idea de que las nubes tienen una naturaleza gaseosa, en lugar de considerar que están constituidas por pequeñas gotas de agua en estado líquido, o incluso incurren en el error de creer que la condensación se debe a un descenso de la temperatura. Por tanto, no son capaces de vincular sus explicaciones a fenómenos naturales como la formación de las nubes, el vaho en invierno, la niebla, el rocío, etc. Asimismo, otros estudios como los de Ben-Zvi-Assaraf y Orion (2005), señalaron que las ideas erróneas sobre procesos físico-químicos básicos como la evaporación, la condensación y la disolución se mantienen durante toda la Educación Secundaria.

Por su parte, Castelltort y Sanmartí (2013), también analizaron las dificultades que tienen los estudiantes del segundo y tercer ciclo EP para representar el ciclo natural y urbano del agua. Respecto del ciclo natural, los resultados mostraban que el alumnado no ilustra el cierre del ciclo y solo consideraba la circulación atmosférica, sin tener en cuenta la circulación subterránea. De igual forma, tampoco representaban el ciclo urbano, ni una red de transporte y tratamiento de agua -sin diferenciar potabilización y depuración-. Estas autoras advierten sobre la necesidad de introducir una visión integral de ambos ciclos, y no, como escenarios independientes. También, hay que destacar que cuando los estudiantes representaban el ciclo completo, en ocasiones lo hacían desde una perspectiva antropocéntrica, ilustrando elementos tales como depuradoras, conducciones de agua, alcantarillas, niños bebiendo agua, piscinas y duchas, grifos o fuentes de los que aflora agua (Martín del Pozo 2013).

Estudios más recientes apuntan en el mismo sentido y siguen mostrando las dificultades del alumnado en cuanto a los conceptos asociados al agua. Así, Amahmid et al. (2019) revelan que son pocos los estudiantes que responden correctamente a preguntas sobre el ciclo del agua, su composición química o aspectos relacionados con el saneamiento. Por su parte, HavuNuutinen, Kärkkäinen y Keinonen (2018), encontraron que el alumnado de $4^{\circ}$ y $5^{\circ}$ de EP tiene la capacidad de identificar el agua como compuesto químico, pero posee un conocimiento cotidiano sobre sus características, asociadas a menudo al color y olor del agua. Respecto de los estados del agua, los conceptos son empleados de forma confusa y el alumnado solo justifica los cambios de estado al relacionarlos con el clima y las estaciones. También, indican que los estudiantes tienen problemas para definir el agua superficial o subterránea. Resultados similares fueron obtenidos por Basterretxea et al. (2019) que comparando varias generaciones de estudiantes (1996-2013), concluyeron que el ciclo del agua solo es percibido desde la perspectiva de algunas propiedades del agua (derretir, helar, llover, nevar, ...), sin considerar otros aspectos relacionados como las plantas, el suelo o los animales, fundamentales para entender el funcionamiento de los sistemas de distribución y transporte de materia dentro del ciclo del agua. Además, estos autores indicaron que habían aumentado las referencias a elementos del ciclo urbano (tuberías, depósitos, ...) en un $60 \%$, y solo una cuarta parte de los estudiantes analizados hacía referencia o explicaba los procesos que intervienen en el tratamiento del agua (potabilización y depuración). En este sentido, estos autores 
destacan un incremento en el desconocimiento de la relación existente entre el ciclo del agua y su recorrido urbano entre los estudiantes de 1996 y 2013.

Algunos autores sostienen que esta falta de conocimiento y de adquisición de conceptos erróneos está ligada al enfoque de desconexión con sus experiencias vitales cotidianas que se producen en las aulas (Fernández-Ferrer, González García y Carrillo-Rosúa 2008; Liefländer, Fremerey y Bogner 2016). Además, el tradicional tratamiento descriptivo de este contenido en la enseñanza de las ciencias tampoco permite abordarlo desde su complejidad (Aragüés Díaz 2019; Díez 2017). También, otros autores señalan que la televisión, internet, los libros de texto, o la falta de especialización docente pueden ser fuentes de confusión (De Miguel et al. 2009). En este sentido, Reyero et al. (2007) sostienen que las ilustraciones del ciclo del agua encontradas en distintas fuentes bibliográficas que mostraban "el viaje de una gota" siempre en estado líquido, es un elemento favorecedor en la adquisición de estas ideas y conceptos erróneos.

Por todo ello, se considera necesario seguir profundizando en la comprensión sobre la manera en la que los estudiantes construyen y consolidan su conocimiento sobre el concepto del agua (Basterretxea et al. 2019).

\section{El concepto del agua como contenido curricular en la Educación Primaria}

Algunos estudios indican que los contenidos curriculares que han cobrado más importancia dentro del concepto de agua son los referidos a las características y propiedades del agua, su ciclo natural, el papel del agua en la configuración del paisaje, etc. (Márquez y Bach 2007; Bar 1989; Cuello y Navarrete 1993; Marcén 2010). No obstante, en los últimos años ha crecido el interés por abordar otros aspectos referidos a los principales usos del agua o las problemáticas socioambientales vinculadas al agotamiento y contaminación de este recurso (Amahmid et al. 2019; Fernández y Rodríguez 2017; Havu-Nuutinen, Kärkkäinen y Keinonen 2018; Moreno Fernández 2017). En este sentido, Cañal, García-Carmona y Cruz-Guzmán (2016), por ejemplo, indican la importancia de que los estudiantes tomen conciencia de la contaminación no solo desde una perspectiva macroscópica (basuras), sino también considerando las causas y los responsables de esta.

Sin embargo, no parece ser ésta la línea que se recoge en el currículo de EP (Gobierno de España 2015). Así, Martínez et al. (2020), que realizaron una revisión sobre la importancia que se le concede a los conceptos del agua en el currículo español de EP, pusieron de manifiesto que existen pocas referencias en el currículo educativo sobre el concepto del agua. Sus resultados muestran que el contenido del agua es tratado desde las asignaturas de Ciencias Naturales y Ciencias Sociales desde un enfoque en el que se prima lo teórico, en comparación con la perspectiva de adquirir conductas y actitudes de sostenibilidad abordadas en términos de consumo y uso racional del agua.

Resultados similares se han podido constatar al revisar el tratamiento que se hace sobre el concepto del agua en la normativa educativa andaluza de EP (Junta de Andalucía 2015). El análisis ha puesto de manifiesto que, aunque se realizan diversas referencias a conceptos sobre el agua a largo de los tres ciclos de EP, sigue primando lo teórico frente a lo conductual y actitudinal. Así, en el primer ciclo, se recoge el contenido del agua como un recurso fundamental del medio físico, siendo las temáticas que deben abordarse: las propiedades del agua, los cambios de estado, el ciclo del agua, sus diversos usos, algunas problemáticas ambientales como la sequía o la contaminación derivada de la actividad humana, y la importancia de cuidarla y conservarla por considerarse un recurso vital. El segundo ciclo se enfoca en las interacciones entre el agua, el ser humano y la naturaleza. En esta etapa se amplía 
el conocimiento de las características del agua y se profundiza en la descripción y diferenciación de los cambios de estado observados desde lo cotidiano y lo natural. El ciclo del agua se aborda integrando diferentes elementos y procesos hídricos (disolución, evaporación, filtración, precipitaciones, ...), y también se estudian las características y composición de la hidrosfera (masas de agua y su distribución) junto al funcionamiento de los ecosistemas acuáticos. Por ejemplo, se introduce el concepto de aguas subterráneas y su configuración, su forma de aflorar y las posibilidades de acceder a ellas. Además, se identifica el agua como un recurso agotable y se promueve su uso responsable. En el tercer ciclo, se explica el ciclo del agua de manera integral, pudiéndose reconocer componentes y sistemas más complejos y abstractos (relieve, erosión, clima, ...). También se relaciona la importancia del agua con los factores de deterioro y regeneración, siendo la contaminación, el derroche y el desarrollo de actuaciones conceptos principales para su conservación. Igualmente, adquiere especial relevancia en este ciclo abordar el tema de las energías renovables y no renovables en relación al agua, así como los beneficios y los riesgos de su utilización: agotamiento, lluvia ácida, etc.

Teniendo en cuenta este marco referencia, se plantea un estudio dirigido a conocer y analizar los conceptos científicos de los estudiantes del tercer ciclo de EP, sobre los procesos naturales, los usos y gestión del agua y, las problemáticas derivadas de la escasez y contaminación de este recurso. Este trabajo recoge investigaciones previas y nuestros propios hallazgos para proporcionar una base empírica sobre la comprensión de los estudiantes acerca de algunas concepciones sobre el agua. También se muestran algunos de los desafíos y dificultades que enfrentan los estudiantes en la adquisición y desarrollo de estos conocimientos. Esto permitirá disponer de información para diseñar propuestas didácticas que actúen tanto en la detección de lagunas, errores o inconsistencias en el aprendizaje sobre esta temática, como que ayuden en la construcción de un conocimiento adecuado sobre la misma.

\section{Metodología}

\section{Muestra}

Se aplicó un muestreo teórico y motivado de carácter cualitativo (Tójar 2006), atendiendo a las características relevantes que son esenciales para la investigación. La muestra estuvo compuesta por las producciones (que se detallarán más adelante) de 95 estudiantes de tercer ciclo de EP con edades comprendidas entre los 10 y 12 años, de los cuales el 44,2\% eran chicas y 55,8\% chicos. Los que pertenecían al inicio del $5^{\circ}$ curso suponían un total de 47 estudiantes (49,5\%), de los cuales, 29 eran niños y 18 niñas. El $6^{\circ}$ curso lo conformaban 48 estudiantes $(50,5 \%)$, de los cuales, 24 eran niños y 24 niñas. Los estudiantes pertenecían a un centro educativo concertado en una zona de nivel socioeconómico medio-bajo de la ciudad de Málaga. A cada escolar se le pidió dos producciones, por lo que se contó con 190 documentos de diversa naturaleza, pero sobre el mismo tema principal, para su análisis primario cualitativo.

\section{Enfoque metodológico, procedimientos y análisis de datos}

Esta investigación se realizó desde un enfoque mixto, con un diseño CUAL $\rightarrow$ cuan, esto es, método primario cualitativo, que condujo a un análisis complementario secundario cuantitativo (Pereira 2011). Para recoger y analizar la información del estudio, se desarrolló una estrategia secuencial exploratoria. Este proceso consistió en realizar un análisis de las cartas y dibujos a través de la construcción de un sistema de categorías, que permitió obtener un conjunto de datos cualitativos agrupados y clasificados (unidades de análisis) en función de distintos criterios temático. Posteriormente, se transformaron y organizaron estas unidades de análisis en matrices descriptivas (Miles, Huberman y Saldaña, 2014), para ser analizadas mediante estadística descriptiva básica (frecuencias y porcentajes). Por consiguiente, sobre un 
primer análisis cualitativo de las cartas y dibujos, se realizó un análisis secundario cuantitativo de frecuencias y porcentajes, que evidenció de manera complementaria la mayor presencia, o ausencia, de algunas de las categorías.

Como instrumentos de recogida de datos se usaron producciones creativas requeridas a los participantes: cartas y dibujos. Este tipo de producciones son usadas para recoger los conocimientos que reflejen de manera indirecta la concepción de jóvenes y estudiantes sobre determinados fenómenos, y poder realizar análisis cualitativos en profundidad (Paula y Borges 2019; Jiménez-Yañez, Mancinas y Martínez-Soto 2008). Ambas producciones fueron realizadas por los escolares, a los cuales se les presentó una situación imaginaria en la que debían explicar y describir a unos seres extraterrestres, qué interés y relevancia tenía para ellos el agua en nuestro planeta. Para promover la reflexión y el pensamiento creativo de los estudiantes se formularon las siguientes cuestiones: ¿Qué es el agua?, ¿Para qué se utiliza?, ¿Dónde la encontramos?, ¿En qué procesos intervienen?, etc.

En el análisis de los datos extraídos de las 95 cartas y 95 dibujos de los estudiantes, se desarrollaron procesos de categorización, interpretación y elaboración de conclusiones (Tójar 2006). Como apoyo al análisis cualitativo se empleó el programa Atlas.ti v7.0 (2012). En concreto, se elaboró un sistema de categorización mixta, a partir de un proceso de categorización inductiva -análisis del contenido de cartas y dibujos-, y deductivas -análisis a partir de modelos teóricos- (Márquez y Bach 2007; Martín del Pozo 2013; Martínez et al. 2020), mediante tareas interconectadas de reducción, disposición de los datos, transformación, extracción y verificación de la información obtenida. Para ello, se utilizaron técnicas como la clasificación y la categorización, modelos y tipologías. En este sentido, los datos recogidos adquirieron cuerpo de teoría, a través de un proceso de abstracción creciente (Llosa 2014). Una vez organizada la información, se construyeron gráficas y matrices explicativas para establecer conexiones entre los datos y realizar interpretaciones sobre los mismos (Miles, Huberman y Saldaña 2014). Seguidamente, se realizaron procesos de extracción y revisión de la información que permitieron identificar regularidades, construir marcos de referencia y elaborar tipologías de datos. De las transcripciones realizadas, se seleccionaron las citas textuales y los dibujos más relevantes para crear el sistema de macrocategorías. En este trabajo se presenta una de las macrocategorías obtenidas, denominada "Procesos naturales, gestión y problemáticas del agua". Esta contiene 3 categorías y 20 subcategorías relacionadas con los conocimientos científicos del alumnado, sobre el origen y las fases de creación del ciclo hidrológico (natural y urbano), y los problemas de contaminación y escasez del agua.

\section{Resultados}

Para la construcción de la macrocategoría "Procesos naturales, gestión y problemáticas del agua" se seleccionaron 268 citas literales de las cartas de un total de 1.167, y 138 fragmentos de 95 dibujos diferentes, que permitieron definir 3 categorías: categoría 1.1"Procesos naturales del agua", categoría 1.2 "Gestión y usos del agua" y categoría 1.3 "Problemáticas del agua: Escasez y contaminación”.

La tabla 1 muestra las referencias realizadas en las cartas y los dibujos de los estudiantes de EP sobre los conceptos relacionados con los procesos naturales del agua (categoría 1.1). Como se puede observar, los conocimientos relacionados con la distribución superficial del agua en la hidrosfera son los más aludidos (subcategoría 1.1.2) (90,7\%), seguidos de aquellos relacionados con los cambios de estado del agua (subcategoría 1.1.4) (82,1\%):

"Hay dos tipos de agua: Las aguas continentales se pueden encontrar en lagos, ríos, charcos y muchísimos más, y las marinas se encuentran en océanos y mares" C105CI (10:7) 
"El agua se encuentra normalmente en estado líquido, pero si el agua se calienta cambia de estado a gaseoso y si lo enfrías cambia a estado sólido" C106CI (10:4)

Estos resultados ilustran que la mayoría de los participantes parece tener asimilados los conocimientos sobre las distintas masas de agua (tanto naturales como artificiales), y los cambios de estado (líquido, sólido y gaseoso), pertenecientes al primer ciclo de EP. Por el contrario, los conceptos menos referidos son los relacionados con el proceso del ciclo del agua (subcategoría 1.1.6) (6,3\%) y el dinamismo de las fases del ciclo (subcategoría 1.1.7) (5,3\%), que corresponden al conocimiento que tienen los estudiantes sobre el carácter cíclico, constante y cerrado del ciclo del agua.

Tabla 1. Frecuencias y porcentajes de las citas textuales y fragmentos de dibujos de la categoría "Procesos naturales del agua (ciclo hidrológico natural") ${ }^{1}$

\begin{tabular}{|c|c|c|c|c|}
\hline Macrocategoría 1. Procesos naturales, gestión y problemáticas del agua & \multicolumn{2}{|c|}{ Cartas } & \multicolumn{2}{|c|}{$\begin{array}{c}\text { Fragmentos de } \\
\text { dibujos }\end{array}$} \\
\hline Categoría 1.1. Procesos naturales del agua (ciclo hidrológico natural) & $\mathrm{f}$ & $\%$ & $\mathrm{f}$ & $\%$ \\
\hline Subcategoría 1.1.1 Propiedades físicas y eléctricas & 43 & 45,3 & 1 & 1,1 \\
\hline Subcategoría 1.1.2. Distribución superficial del agua en la hidrosfera & 90 & 90,7 & 27,4 & 18,8 \\
\hline Subcategoría 1.1.3. Distribución subterránea del agua en la hidrosfera & 17 & 17,9 & 2 & 2,1 \\
\hline Subcategoría 1.1.4. Cambios de estado del agua & 78 & 82,1 & 1 & 1,1 \\
\hline Subcategoría 1.1.5. Origen de las fases del ciclo del agua & 29 & 30,5 & 13 & 13,7 \\
\hline Subcategoría 1.1.6. Procesos del ciclo del agua & 6 & 6,3 & 12 & 12,6 \\
\hline Subcategoría 1.1.7. Dinamismo de las fases del ciclo del agua & 5 & 5,3 & 2 & 2,1 \\
\hline
\end{tabular}

En relación a los fragmentos de dibujos que aparecen en la tabla 1, la subcategoría más representada vuelve a ser la relacionada con los conceptos sobre la distribución superficial del agua en la hidrosfera (subcategoría 1.1.2) (18,8\%). Como se observa en el ejemplo de la figura 1 , esta subcategoría se ve reflejada porque los estudiantes dibujan un planeta casi completamente azul y diferentes escenarios naturales (como un valle o montañas) donde se observan grandes masas de agua que ocupaban todo el dibujo, como ríos, lagos, mares, etc. Respecto de las subcategorías menos representadas son las referidas a las propiedades físicas y eléctricas $(1,1 \%)$ y los cambios de estado del agua (1,1\%), subcategorías 1.1.1 y 1.1.4 respectivamente. Resulta llamativa la baja representatividad de estos contenidos en los dibujos, considerando que son conceptos que se trabajan al inicio de la EP, y que fueron bastante aludidos en las cartas.

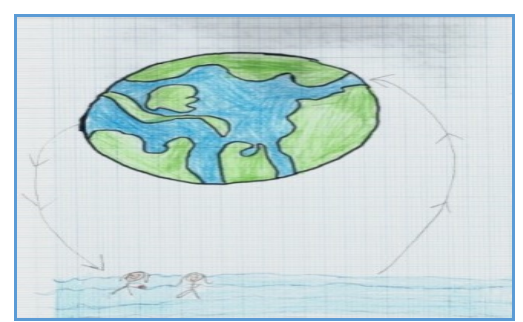

Figura 1. Código del dibujo: D106CI

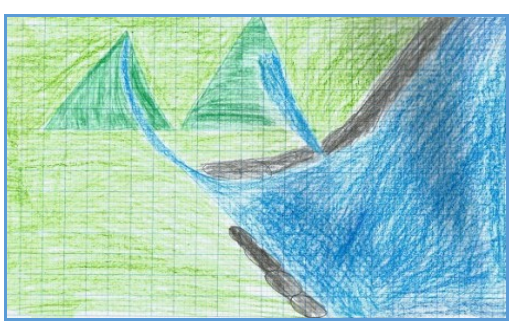

Figura 2. Código del dibujo: D115XI

Figuras 1 y 2. Ejemplos de la subcategoría "Distribución superficial del agua en la hidrosfera".

En relación a la categoría 2.1 sobre la gestión y usos del agua (Tabla 2), se puede observar que, el mayor número de referencias en las cartas se relaciona con la microcategoría consumo para higiene personal $(71,6 \%)$ y consumo para ocio $(52,5 \%)$ :

${ }^{1}$ El porcentaje (\%) no suma 100 debido a la no exclusividad mutua. 
"En las tiendas y bazares venden botellines de agua para que los compres y te los bebas" (C026XI, 02:14).

"El agua es muy necesaria para ducharnos, para hacer de comer, para lavarnos la cara, para lavarnos los dientes" (C045CI, 04:01).

Tabla 2. Frecuencias y porcentajes de las citas textuales y fragmentos de dibujos de la categoría "Gestión y usos del agua"2

\begin{tabular}{|c|c|c|c|c|}
\hline Macrocategoría 1. Procesos naturales, gestión y problemáticas del agua & \multicolumn{2}{|c|}{ Cartas } & \multicolumn{2}{c|}{$\begin{array}{c}\text { Fragmentos de } \\
\text { dibujos }\end{array}$} \\
\hline Categoría 2.1. Gestión y usos del agua & $\mathrm{f}$ & $\%$ & $\mathrm{f}$ & $\%$ \\
\hline Subcategoría 2.1.1. Captación & 5 & 5,3 & 2 & 2,1 \\
\hline Subcategoría 2.1.2. Transporte y almacenamiento & 2 & 2,1 & 1 & 1,1 \\
\hline Subcategoría 2.1.3. Potabilización & 25 & 26,3 & 2 & 2,1 \\
\hline Subcategoría 2.1.4. Distribución (Alcantarillado, Retorno y Grifo) & 18 & 18,9 & 5 & 5,3 \\
\hline Subcategoría 2.1.5. Consumo & $\mathrm{f}$ & $\%$ & $\mathrm{f}$ & $\%$ \\
\hline Microcategoría 2.1.5.1. Consumo para alimentación & 18 & 18,9 & 27 & 28,4 \\
\hline Microcategoría 2.1.5.2. Consumo para higiene personal & 68 & 71,6 & 8 & 8,4 \\
\hline Microcategoría 2.1.5.3. Consumo doméstico & 40 & 42,1 & 3 & 3,2 \\
\hline Microcategoría 2.1.5.4. Consumo para ocio & 50 & 52,6 & 26 & 27,4 \\
\hline Microcategoría 2.1.5.5. Consumo para protección y seguridad & 1 & 1,1 & 0 & 0 \\
\hline Subcategoría 2.1.6. Tratamiento/Depuración & 6 & 6,3 & 1 & 1,1 \\
\hline
\end{tabular}

En contraposición, los conceptos a los que menos aluden están también relacionados con el consumo, pero en este caso para protección y seguridad $(1,1 \%)$.

Respecto de los contenidos referidos al recorrido urbano del agua, el mayor número de referencias en las cartas se observan de la subcategoría 2.1.3 sobre la potabilización del agua $(26,3 \%)$, siendo lo menos referido las ideas relacionadas con la captación del agua (subcategoría 2.1.1) (5,3\%) y el transporte y almacenamiento de la misma (subcategoría 2.1.2) $(2,1 \%)$. Estos últimos son mencionados de forma puntual y aislada a partir de algún elemento relacionado con tuberías, alcantarillas o grifos.

Resultados similares a estos se obtienen en los fragmentos de dibujos (Tabla 2), dado que lo que menos pintan son los contenidos relacionados con el recorrido urbano del agua, concretamente, los que tienen que ver con la captación $(2,1 \%)$, el transporte y almacenamiento $(1,1 \%)$, y el tratamiento y depuración $(1,1 \%)$. Hay que señalar que no se esperaban porcentajes altos en estas subcategorías porque estos conceptos no empiezan a ser trabajados hasta el último curso de EP. Por otro lado, las subcategorías más representadas, al igual que en las cartas, son la de consumo pero en este caso, relacionados con la alimentación (microcategoría 2.1.5.1) $(28,4 \%$ y con el ocio (microcategoría 2.1.5.4) (27,4\%). Como se observa en la figura 4, los estudiantes representan el consumo para alimentación dibujando principalmente a personas bebiendo agua o envases llenos de agua como botellas o garrafas; y el consumo para el ocio lo representan a través de actividades relacionadas con el mar como pescar, nadar, bucear, etc., como se puede apreciar en la figura 3.

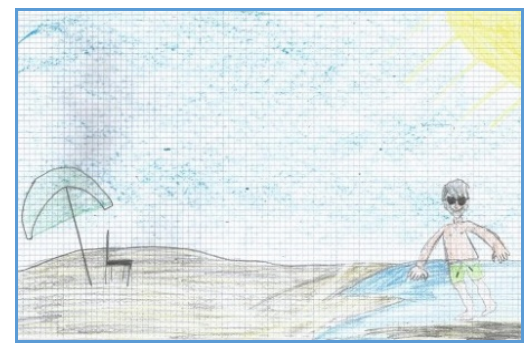

Figura 3. Código del dibujo: D075XI

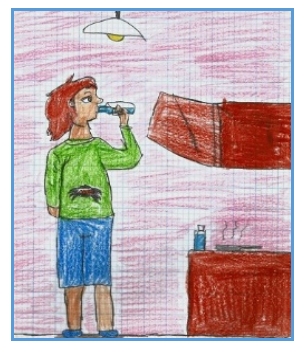

Figura 4. Código del dibujo: D245XI

${ }^{2}$ El porcentaje (\%) no suma 100 debido a la no exclusividad mutua. 
En relación a los conceptos relacionados con las problemáticas del agua: escasez y contaminación (categoría 3.1), en general, la tabla 3 muestra cómo las referencias a estos son muy escasas. De manera más específica se observa que el mayor número de referencias, corresponde a la categoría sobre el consumismo (categoría 3.1.1), tanto en los dibujos (3,2\%), como en las citas de las cartas (7,4\%): "Hay también muchas personas las cuales dicen que sí se puede vivir sin agua, pero tenemos que comprar garrafas de agua en algún supermercado (Mercadona, Dia) pero ¿Cuántas garrafas de agua deberías comprar a la semana, al mes, incluso al año para poder vivir?” C156XI (15:6).

Tabla 3. Frecuencias y porcentajes de las citas textuales y fragmentos de dibujos de la categoría "Problemáticas del agua"3

\begin{tabular}{|l|c|c|c|c|}
\hline Macrocategoría 1. Procesos naturales, gestión y problemáticas del agua & \multicolumn{2}{|c|}{ Cartas } & \multicolumn{2}{c|}{$\begin{array}{c}\text { Fragmentos de } \\
\text { dibujos }\end{array}$} \\
\hline Categoría 3.1 Problemáticas del agua: Escasez y contaminación & $\mathrm{f}$ & $\%$ & $\mathrm{f}$ & $\%$ \\
\hline Subcategoría 3.1.1. Consumismo (agricultura, industria, uso urbano, ...) & 7 & 7,4 & 3 & 3,2 \\
\hline Subcategoría 3.1.2. Sobreexplotación de acuíferos & 0 & 1,1 & 1 & 1,1 \\
\hline Subcategoría 3.1.3. Desertificación & 2 & 2,1 & 0 & 0 \\
\hline Subcategoría 3.1.4. Pérdidas/desperdicio de agua & 3 & 3,2 & 1 & 1,1 \\
\hline Subcategoría 3.1.5. Contaminación por vertidos urbanos & 5 & 5,3 & 2 & 2,1 \\
\hline
\end{tabular}

Asimismo, se siguen encontrando coincidencias entre lo que describen y lo que dibujan, siendo en ambos casos, la desertificación (cartas: $2,1 \%$, dibujos: $0 \%$ ) y la sobreexplotación de los acuíferos (cartas y dibujos: 1,1\%) las subcategorías a la que menos aluden. En cuanto a la desertificación, parece que el alumnado aún no es muy consciente de las causas de esta problemática ambiental, aunque aparecen menciones en algunas cartas sobre algunas de las consecuencias relacionadas con la supervivencia de los organismos o las plantas: "Si no hubiera agua no habría vegetación, no habría alimentos. En fin, sería una catástrofe, el planeta sería un desierto" C165CI (16:4).

Resulta sorprendente que la categoría sobre las pérdidas/desperdicio de agua tenga una representatividad tan baja tanto en las cartas $(3,2 \%)$ como en los dibujos $(1,1 \%)$, siendo un contenido esencial que comienza impartiéndose en Educación Infantil y, se amplía durante toda la EP. También, hay que destacar la gran cantidad de recursos, iniciativas y campañas de sensibilización que han surgido en las últimas décadas dirigidas a informar y concienciar a la población sobre las causas y los efectos del derroche del agua en nuestro país, que parecen no haber tenido el impacto esperado en los participantes.

A continuación, se presentan algunos ejemplos de citas, fragmentos de dibujos y códigos de la macrocategoría "Problemáticas del agua derivadas de su escasez y contaminación" (Tabla 4). Se ha seleccionado esta tabla para ilustrar el nivel de conciencia socioambiental que presenta el alumnado en relación con el conocimiento sobre el impacto de la actividad humana en el cuidado y conservación del agua. Resulta de interés y relevancia dar visibilidad a la importancia que tiene trabajar estos contenidos dentro del aula para construir una ciudadanía crítica, consciente y responsable con su estilo de vida y hábitos de consumo.

Para este estudio, las denominaciones de "familia de códigos" y "códigos" que utiliza el programa Atlas.ti, se han cambiado a "categorías", para las primeras, y "subcategorías", para las segundas. La denominación de "códigos" se ha utilizado como etiquetas de las subcategorías.

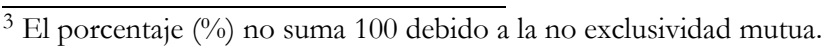


Tabla 4. Citas y fragmentos de dibujos de la categoría. Problemáticas del agua: Escasez y contaminación del agua

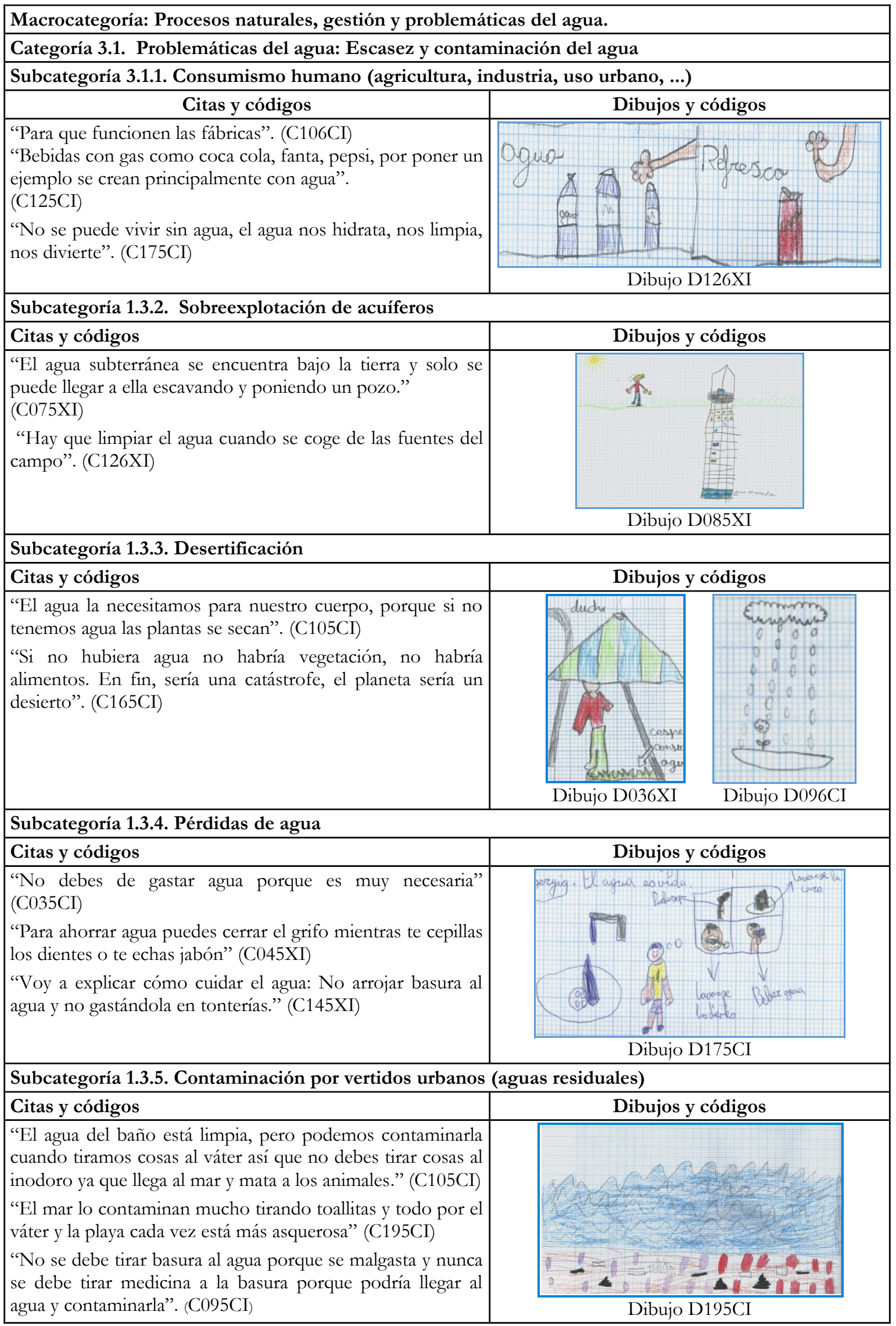




\section{Conclusiones}

El análisis de las cartas y los fragmentos de los resultados de este estudio muestran que el alumnado tiene un conocimiento amplio sobre la distribución superficial del agua en la hidrosfera. Según Cuello y Navarrete (1992) existe una clara progresión desde las concepciones iniciales de los niños más pequeños, que consideran los ríos como cauces simples, hasta la consideración de una cuenca hidrográfica más compleja en relación primero con masas de agua superficiales, y después con subterráneas. Esta evolución puede deberse a que se trabaja más y se le da mayor importancia al concepto de agua superficial que al de subterránea, especialmente en los libros de texto (Fernández-Ferréz et al. 2008). En este sentido, como muestran los resultados de este estudio, el alumnado participante identifica los diferentes lugares donde se pueden encontrar masas de aguas superficiales (ríos, lagos, mares, océanos, etc.), y en muy poca medida las subterráneas. Ambos son un conocimiento relevante para llegar a la adquisición del concepto de captación. En relación a las superficiales, resulta llamativo que incluyan el concepto "charco" como agua superficial dentro del ciclo del agua, entendiendo que el agua no solo se presenta en ambientes naturales como grandes masas de agua. Del mismo modo, de acuerdo con los resultados de Guerrero Valle (2017), parece que el alumnado participante olvida que cuando llueve, el agua no solo se deposita en el medio natural (charcos, debajo del suelo, ...), sino también en el medio urbano (tubería, casa, suelo, la ciudad, ...).

Respecto de las aguas subterráneas, diversos autores advierten sobre la existencia de errores conceptuales y dificultades en la enseñanza y el aprendizaje sobre este concepto, subrayando la necesidad de construir recursos o modelos tangibles que simulen la realidad y permitan clarificar ideas básicas respecto del mismo (Bar 1989; Massa 1994; Ben-Zvi-Assaraf y Orion 2005; Dickerson y Dawkins 2004; Guerrero Valle 2017, entre otros). Estos recursos ayudarían al alumnado a desechar ideas erróneas como, por ejemplo, algunas de las obtenidas en este trabajo como que los únicos afloramientos espontáneos de las aguas subterráneas son las fuentes; como que el agua subterránea se encuentra a gran profundidad y su única salida a la superficie es a través de pozos; o como que los acuíferos son una especie de depósito o embalse de agua bajo tierra constituidos como una reserva de agua aislada, sin conexión con el ciclo hidrológico. En este sentido, nuestros hallazgos coinciden con los trabajos de Hernández Arnedo (2013), Santana, Cabrera y Pérez (2015) y Unterbruner, Hilberg y Zwischen (2016), que también encontraron que estudiantes de Educación Secundaria creen que el agua subterránea solo se acumula en pozos y cuevas subterráneas, y que esta no está integrada dentro del ciclo del agua ni tampoco tiene relación alguna con las aguas y rocas del entorno. Estos resultados ponen de manifiesto la dificultad que podría llegar a tener el alumnado para adquirir conocimiento y sensibilizarse ante las problemáticas ambientales sobre la sobreexplotación de los acuíferos por la extracción de agua de los pozos, y con ello, los efectos de la actividad humana (uso, gestión y contaminación) en el ciclo hidrológico (Ben-ZviAssaraf y Orion 2005).

En relación a los procesos del ciclo del agua, como se muestran en los resultados, el alumnado participante representa el ciclo del agua de manera errónea, incompleta o difusa. Así, estos describen con cierta facilidad los cambios de estados, aunque no son capaces de ilustrar de manera práctica todos los procesos que intervienen en el ciclo del agua, exceptuando el proceso de precipitación. Solo algunos estudiantes mencionan en sus cartas el proceso de evaporación de manera explícita, ocurriendo algo similar en los dibujos de quienes representan el proceso con unas flechas ondulantes dirigidas hacia el cielo. Es destacable que ningún estudiante hace alusión a ninguna fuente de energía (sol), ni a determinados aspectos relacionados con los procesos de evaporación y precipitación. Así, en el caso de la evaporación 
del agua solo lo consideran en los mares, océanos o ríos, desechando en la mayoría de las ocasiones la idea de que pueda suceder desde las plantas, el suelo (ej. charcos), superficies líquidas, animales, moléculas de agua expulsadas de nuestro cuerpo, etc. (Basterretxea et al. 2019). De igual forma, el alumnado implicado en el estudio no relaciona entre el proceso de condensación y la formación de las nubes, igual que ocurre en otros trabajos como el de Guerrero Valle (2017). Asimismo, no mencionan el dinamismo de los elementos y procesos que intervienen en los ecosistemas y los sitúan en un continuo unidireccional (Díez 2017). Esta circunstancia puede tener su explicación dado que la mayoría de los niños y niñas de EP tienen dificultades para entender la dinámica cíclica. Muchos piensan que hay un principio (en la formación de las nubes) y un fin (el retorno de las aguas superficiales a los mares y océano) dentro del ciclo (Hernández Arnedo 2013). Además, según estos autores, estas ideas pueden llevar a pensar al estudiante que la cantidad del agua del mar aumentará con el tiempo por el aporte de los ríos y que si se produce un incremento de población aumentará el consumo, disminuyendo sin ningún tipo de problema la cantidad total de agua del sistema. Por tanto, al igual que se ha dicho anteriormente, esto puede tener repercusión a la hora de que los estudiantes entiendan las problemáticas medioambientales que se derivan del uso que el ser humano hace del agua.

Respecto de los conceptos sobre la gestión y uso del agua, los estudiantes participantes en este trabajo hacen referencias a los conceptos de consumo, especialmente, al consumo para higiene personal y ocio. Aunque, parecen tener menos conocimientos de los procesos que intervienen en el recorrido urbano del agua como la captación, transporte y saneamiento. Resultados similares encontraron Covitt, Gunckel y Anderson (2009), dado que en su estudio mostraron cómo los estudiantes de EP tenían concepciones científicas confusas y erróneas sobre los elementos y las conexiones de los sistemas del agua, así como de los sistemas de tratamiento del agua. Esta circunstancia apunta a la dificultad que podrían tener los estudiantes para vincular el agua con el cuidado del medio ambiente y los motivos de la contaminación (Sari, Karkkainen y Tuula 2018), y que se corrobora con los resultados de este trabajo dada la baja referencia de los participantes a las problemáticas del agua como la escasez o la contaminación.

En definitiva, esta falta de conocimiento sugiere que el sistema educativo contribuye de manera escasa al desarrollo de una ciudadanía bien informada sobre la procedencia y destino del agua potable, su impacto directo en el ciclo del agua y en la responsabilidad sobre su contaminación y conservación (Díez 2019). En este sentido, algunos autores (Amahmid et al. 2019; Zhan, He y Wing Mui So 2019) sugieren aplicar programas educativos que no solo se dirijan a la adquisición de conocimiento científico, sino también al desarrollo de habilidades, actitudes y comportamientos sobre las problemáticas del agua. Es decir, desde las aulas se debe potenciar que el alumnado adquiera ciertas habilidades que les permitan utilizar el conocimiento científico asociado al agua para poder actuar ante su problemática.

Por ello, se propone que desde la práctica educativa se trabaje a partir de enfoques interdisciplinares y constructivistas en torno a problemáticas sociocientíficas concretas asociadas al agua. Enfoques que promuevan la puesta en práctica de métodos innovadores centrados en el aprendizaje de los estudiantes y lo más contextualizadas en su ámbito cotidiano. Ejemplo de ello podría ser el diseño de propuestas de enseñanza centradas en el consumo de agua embotellada, como la presentada por Rodríguez-Mora, Cebrián-Robles y Blanco-López (2021), que aunque dirigida a Educación Secundaria, puede servir de base y punto de partida para el diseño y puesta en práctica de propuestas educativas de esta temática para el tercer ciclo de Educación Primaria. Con ellas podrían abordarse diferentes conocimientos científicos asociados a la contaminación que además también proponemos pasen por conocer el origen, la dinámica, el recorrido y el tratamiento del agua. En este 
sentido, se propone como guía que estas propuestas se trabajen a partir de las ideas recogidas por Castelltort y Sanmartí (2016) para orientar la formación de la cultura ambiental del agua de los estudiantes.

Se sugiere además que estas propuestas y, por tanto, estos conocimientos se trabajen a partir de la argumentación y la toma de decisiones para propiciar que los estudiantes desarrollen diferentes habilidades como el pensamiento crítico. Para ello, se recomiendan estrategias metodológicas para el profesorado consistentes en: fomentar el debate sobre los contenidos de la problemática del agua propuesta a partir de preguntas que cuestionen los argumentos de los estudiantes; potenciar el uso de datos como pruebas en los argumentos aportados; y propiciar la valoración de diferentes alternativas proporcionando argumentos alternativos. Con ello, además se estará promoviendo, tal y como Andreucci-Annunziata (2016) recomienda, un contexto de aprendizaje focalizado en la interacción entre todos los participantes implicados en el proceso de enseñanza-aprendizaje, alumnado y profesorado.

Los resultados de este trabajo muestran las dificultades y desafíos del aprendizaje de los conceptos relacionados con el agua en la última etapa de la EP, poniendo en valor la necesidad de conocer los conocimientos científicos de los estudiantes para el diseño de modelos educativos desde una perspectiva científica y sostenible. Además, se realizan algunas recomendaciones y sugerencias para trabajar la temática del agua en las aulas. También, este trabajo pretende animar a todos los sectores de nuestra sociedad (docentes, familias, profesionales del agua, medios de comunicación, etc.), a sumar esfuerzos para ayudar a los más jóvenes a identificar los temas relevantes en torno al agua, a proponer soluciones que den respuesta a las problemáticas socioambientales derivadas de los usos del agua y a establecer planes educativos que favorezcan la gestión y el uso responsable de este recurso.

Para finalizar es necesario indicar las limitaciones del trabajo y presentar algunas líneas futuras que se derivan de estas. Cabe desatacar que la muestra se limita a estudiantes provenientes de un solo centro educativo concertado de una zona concreta de la ciudad de Málaga. Este hecho, si bien no afecta a la calidad del análisis realizado, hace que las conclusiones obtenidas deban relacionarse específicamente con el grupo de estudiantes que ha participado en el trabajo. Por ello, se pretende continuar la investigación ampliando la muestra a estudiantes pertenecientes a otros centros educativos. Además, como línea futura también se plantea el diseño y la implementación de propuestas de enseñanza que ayuden a revertir algunas de las carencias que se han detectado en la enseñanza de la temática socioambiental del agua, con el fin de contribuir de esta manera al desarrollo de habilidades, actitudes y comportamientos sobre las problemáticas del agua en los estudiantes y en la sociedad en su conjunto. Por otro lado, también indicar que los conocimientos científicos tratados en este estudio suelen abordarse también en etapas educativas posteriores como es el caso de la Educación Secundaria. Por esta razón, señalar que lo mostrado en este trabajo refleja los conocimientos que un grupo concreto de estudiantes, que cursa segundo ciclo de EP, han logrado construir hasta el momento en relación con la temática del agua. Por tanto, indicar que se entiende que el alumnado continuará desarrollando conocimientos y actitudes respecto al agua en etapas educativas posteriores, y que por ello se propone extender el estudio en dichas etapas.

En resumen, esta investigación, realizada desde un enfoque mixto, ha permitido desentramar en profundidad algunos aspectos relevantes en el conocimiento del alumnado de Educación Primaria en relación a las problemáticas del agua y su gestión sostenible. A partir de los resultados se sugiere, de manera añadida, la propuesta de métodos innovadores que se enfoquen en el alumnado y promuevan el aprendizaje significativo a partir de contextos cercanos y cotidianos de los propios estudiantes de Primaria. 
La investigación, además, pone de manifiesto la utilidad de la estrategia de recogida de datos (cartas y dibujos), y de la metodología mixta empleada, en su conjunto, para afrontar este fenómeno de estudio y conocer ciertas claves educativas que deben ser tenidas en cuenta en la práctica docente.

\section{Referencias bibliográficas}

Andreucci-Annunziata, P. (2016). Talento y argumentación: una alianza dialógica en el aula. Revista Curriculum y Formación del Profesorado, 20 (2), 2-17. Recuperado de https://recyt.fecyt.es/index.php/profesorado/article/view/52088/pdf_5

Amahmid, O., El Guamri, Y., Yazidi, M., Razoki, B., Kaid Rassou, K., Rakibi, Y., Knini, G., El Ouardi, T. (2019). Water education in school curricula: impact on children knowledge, attitudes and behaviours towards water use. Int. Res. Geogr. Environ. Educ., 28, 178-193.

Aragüés Díaz, A. (2019). Análisis del conocimiento sobre el ciclo del agua en dos actividades en Primaria. ReiDoCrea: Revista Electrónica de Investigación y Docencia Creativa, 8, 458-466.

Bar, V. (1989). Children's views about the water cycle. Science education, 73 (4), 481-500.

Basterretxea, G., Bastida, S., Díez, J.R. y Gutiérrez, J.M. (2019). Ideas Previas y Educación Ambiental: Comparación entre las ideas del alumnado de dos generaciones (1996 y 2013). Departamento de Medio Ambiente, Planificación Territorial y Vivienda del País Vasco. Servicio Central de Publicaciones del Gobierno Vasco.

Ben-Zvi-Assaraf, O., y Orion, N. (2005). A study of junior high students' perceptions of the water cycle. Journal of Geoscience Education, 53 (4), 366-373.

Castelltort, A. y Sanmartí, N. (2016). Nuevos retos para educar a favor de una nueva cultura ambiental del agua. Revista educ@rnos. Recuperado de https://revistaeducarnos.com/wp-content/uploads/2017/06/articulo-alba.pdf

Cañal, P, García-Carmona, A. y Cruz-Guzmán, M. (2016). Didáctica de las Ciencias Experimentales en Educación Primaria. Colección Didáctica y Desarrollo. Madrid: Ediciones Paraninfo S.A.

Covitt, B. A., Gunckel, K. L., y Anderson, C. W. (2009). Students' developing understanding of water in environmental systems. The Journal of Environmental Education, 40 (3), 37-51.

Cuello, A. y Navarrete, A. (1993). El agua en la ciudad. Materiales didácticos de Educación Ambiental. Junta de Andalucía. Sevilla: Consejería de Educación y Ciencia de la Junta de Andalucía.

De Miguel, Á., Lado, J. J., Martínez, V., Leal, M., y García, R. (2009). El ciclo hidrológico: experiencias prácticas para su comprensión. Enseñanza de las Ciencias de la Tierra, 17 (1), 78-85.

Dickerson, D., y Dawkins, K. (2004). Eighth grade students' understandings of groundwater. Journal of Geoscience Education, 52 (2), 178-181.

Díez, J. R. (2017). El agua y los ríos. Recursos didácticos para el profesorado (2º ciclo Educación Infantil y $1^{\circ}$ ciclo Educación Primaria). Toledo: Ledoria.

Driver, R., Guesne, E. y Tiberghien, A. (1985). Children's ideas in science. Open University Press: Milton Keynes. Traducida al castellano de P. Manzano, 1989. Ideas científicas en la infancia y la adolescencia. Madrid: MorataNEC.

Durkan, N., Güngör, H., Fetihi, L., Erol, A., y Gülay Ogelman, H. (2016). Comparison of environmental attitudes and experiences of five-year-old children receiving preschool education in the village and city centre. Early Child Development and Care, 186 (8), 1327-1341. 
Fernández-Arroyo, J. y Rodríguez-Marín, F. (2016). Los procesos de enseñanza-aprendizaje relacionados con el agua en el marco de las hipótesis de transición. Revista Eureka sobre Enseñanza y Divulgación de las Ciencias, 14 (1), 227-243.

Fernández-Ferrer, G.; González García, F.; Carrillo-Rosúa, J. (2008, septiembre). Los contenidos relacionados con las aguas subterráneas en los textos de estudio, más allá del modelado kárstico. En: Jiménez Liso, M. R. (Ed.). Ciencias para el mundo contemporáneo y formación del profesorado en didáctica de las ciencias experimentales. Actas de los XXIII Encuentros de Didáctica de las Ciencias Experimentales, Universidad de Almería.

Furió, C., Solbes, J. y Carrascosa, J. (2006). Las ideas alternativas sobre conceptos científicos: tres décadas de investigación. Revista Alambique, 48, 64-77.

Gobierno de España (2014). Real Decreto 126/2014, de 28 de febrero, por el que se establece el currículo básico de la Educación Primaria. Boletín Oficial del Estado, 52.

Guerrero Valle, M. (2017). El conocimiento del ciclo del agua en el segundo ciclo de Educación Primaria. Ikastorratz̧a, e-Revista de didáctica, 17, 20-44.

Havu-Nuutinen, S., Kärkkäinen, S., y Keinonen, T. (2018). Changes in primary school pupils' conceptions of water in the context of Science, Technology, and Society (STS) Instruction. International Research in Geographical and Environmental Education, 27 (2), 18-134.

Hernández Arnedo, M. J. (2013). Proyecto Curricular Investigando Nuestro Mundo (6-12). Investigando la Tierra y el Universo. Sevilla: Díada.

Jiménez-Yañez, C.E., Mancinas, R. y Martínez-Soto, Y. (2008). La sociedad del futuro: Una mirada a través del dibujo infantil. Perspectivas de la Comunicación, 1 (2), 7-16.

Junta de Andalucía (2015). Orden de 17 de marzo de 2015, por la que se desarrolla el currículo correspondiente a la Educación Primaria en Andalucía. Boletín Oficial de la Junta de Andalucía, 60.

Karpudewan, M., Roth, W.M. y Abdullah, M.N.S.B. (2015). Enhancing primary school students' knowledge about global warming and environmental attitude using climate change activities. International Journal of Science Education, 37 (1), 31-54.

Liefländer, A., Fremerey, C. y Bogner, F. (2016). Ecuadorian students' conceptions and personal experience regarding water management issues / Concepciones y experiencias personales de los estudiantes ecuatorianos sobre la gestión del agua. Psyecology, Bilingual Journal of Environmental Psychology, 7 (1), 25-63.

Llosa, S. M. (2014). La reconstrucción de las biografías educativas: un ejercicio de memoria y formación. Revista Horizontes Sociológicos, 3 (5), 126-140.

Marcén, C. (2010). El agua, argumento educativo en la Educación Obligatoria y en el sistema social. Tesis Doctoral. Dpto. Geografía y Ordenación del Territorio, Facultad de Filosofía y Letras, Universidad de Zaragoza.

Malandrakis, G., y Chatzakis, S. (2014). Environmental attitudes, knowledge, and alternative conceptions of primary school children in Greece. Applied Environmental Education and Communication, 13 (1), 15-27.

Martín del Pozo, R. (Coord.). (2013). Las ideas "cientificas" de los alumnos y alumnas de Primaria: tareas, dibujos y textos. Madrid: Universidad Complutense de Madrid.

Márquez, C. y Bach, J. (2007). Una propuesta de análisis de las representaciones de los alumnos sobre el ciclo del agua. Enseñanza de las Ciencias de la Tierra, 15 (3), 280-286. 
Martínez-Borreguero, G., Maestre-Jiménez, J., Mateos-Núñez, M. y Naranjo-Correa, F. L. (2020). An Integrated Model Approach of Education for Sustainable Development: Exploring the Concepts of Water, Energy and Waste in Primary Education. Sustainability, 12 (7), 29-47.

Massa, B. (1994). Alphabétisation en Sciences de la Terre: conceptions et obstacles cognitifs sur le cycle de l'eau à l'École Primaire. In A. Giordan, J.-L. Martinand y D. Raichvarg (Éds.), L'alphabétisation scientifique et technique, Actes des XVI des Journées internationales sur la communication, l'éducation et la culture scientifiques et industrielles, 313-318. Paris: DIRES.

Miles, M. B., Huberman, A. M. y Saldaña, J. (2014). Qualitative Data Analysis: A Methods Sourcebook and The Coding Manual for Qualitative Researchers. Journal Technical Communication Quarterly, 24 (1), 109-112.

Mora, F. (2017). Neuroeducación: solo se puede aprender aquello que se ama. Madrid: Alianza.

Moreno-Fernández, O. (2017). ¿Qué sabes de la contaminación? Estudio de las ideas previas en alumnado de Educación Primaria. Revista Electrónica de Enseñanza de las Ciencias, 16 (3), 502-515.

Pereira, Z. (2011). El método mixto en la investigación en educación: Una experiencia concreta. Revista Electrónica Educare, 15 (1), 15-29.

Paula, E. M. A. T. y Borges, W. Dos S. (2019). Imágenes de profesores para niños y adolescentes: narrativas expresas en el teatro de mamulengos y en cartas. Hachetetepé. Revista Científica de Educación y Comunicación, 19, 103-111.

Reyero, C., Calvo, M., Vidal, M. P., García, E. G., y Gabriel, J. (2007). Las ilustraciones del ciclo del agua en los textos de Educación Primaria. Enseñanza de las Ciencias de la Tierra, 15 (3), 287-294.

Rodríguez-Mora, F., Cebrián-Robles, D. y Blanco-López, A. (2021). An Assessment Using Rubrics and the Rasch Model of 14/15-Year-Old Students' Difficulties in Arguing About Bottled Water Consumption. Research in Science Education, (versión online). Doi: https://doi.org/10.1007/s11165-020-09985-z

Santana, A.I., Cabrera, M.C., y Pérez-Torrado, F.J. (2015). Ideas preconcebidas sobre el ciclo del agua y las aguas subterráneas en la educación secundaria de Canarias. En M.C., Cabrera, T., Cruz-Fuentes, V. Mendoza-Grimón y M. P., Palacios-Díaz. II Workshop Estudio, aprovechamiento y gestión del agua en terrenos e islas volcánicas; Instituto Geológico y Minero de España y AIH-Grupo Español, Las Palmas de Gran Canaria.

Sari, H.N; Karkkainen, S. y Tuula, K. (2018). Changes in primary school pupils' conceptions of water in the context of Science, Technology, and Society (STS) instruction. International Research in Geographical and Environmental Education, 27 (2), 118-134.

Taiwo, A. A., Ray, H., Motswiri, M. J. y Masene, R. (1999). Perceptions of the water cycle among primary school children in Botswana. International Journal of Science Education, 21 (4), 413-429.

Tójar, J. C. (2006). Investigación cualitativa: comprender y actuar. Madrid: La Muralla.

Unterbruner, U, Hilberg, S y Schiffl, I. (2016). Understanding groundwater - students' preconceptions and conceptual change by means of a theory-guided multimedia learning program. Hydrology Earth System Sciences, 20 (6), 2251-2266.

Zhan, Y., He, R. y Wing Mui So, W. (2019) Developing elementary school children's water conversation action competence: a case study in China. International Journal of Early Years Education, 27 (3), 287-305. 